\title{
Spectroscopic and line-narrowing properties of resonant sum and difference frequency generation ${ }^{\text {a) }}$
}

\author{
Bernhard Dick ${ }^{\text {b) }}$ and R. M. Hochstrasser \\ Department of Chemistry, University of Pennsylvania, Philadelphia, Pennsylvania 19104 \\ (Received 4 August 1982; accepted 7 October 1982) \\ Three wave mixing is investigated with respect to its spectroscopic applications under fully resonant \\ conditions. A theoretical study of the appropriate second order susceptibility $\chi^{(2)}$ reveals interesting features \\ both for sum and difference frequency generation. For difference frequency generation two different \\ mechanisms are possible one of which allows the observation of resonances between excited states and the \\ study of dephasing induced coherent emission. The coherence decay rate between the two excited states may \\ be measured and also its pure dephasing rate. The line-narrowing characteristics of the fully resonant $\chi^{(2)}$ are \\ studied with respect to correlation between the inhomogeneous distribution for different levels. The line- \\ narrowing capabilities are found to be partly complementary for the sum-and the two difference frequency \\ resonance configurations. This leads to the suggestion of new line-narrowing techniques which could provide \\ information about type and amount of correlation in inhomogeneously broadened systems.
}

\section{INTRODUCTION}

Spectroscopic interest on molecular excited state dynamics is steadily increasing in recent years. While a detailed knowledge of the level structure of the excited states and their assignments is a prerequisite for such work, with increasing molecular size and excitation energy, the spectra become more and more complex and difficult to interpret. The absorption bands are caused to be diffuse by the occurrence of short relaxation times and the interaction of overlapping electronic manifolds. A major source of perturbation for large molecules in condensed phases results from the interactions with the surrounding solvent or solid state environment. These perturbations lead to both homogeneous and inhomogeneous contributions to the absorption linewidth for a given experimental time scale. Both the homogeneous and inhomogeneous contributions contain interesting information about the moleculeenvironment interaction dynamics. A number of techniques involving coherent transient and frequency domain spectroscopy have been developed to unravel spectra of inhomogeneous systems in gases. ${ }^{1-3}$ Not only do these methods provide otherwise hidden knowledge of the level structure, but they also yield dynamical information from studies of the exposed homogeneous line shapes.

Some line narrowing methods require the molecules to be in the gas phase, like saturation spectroscopy, ${ }^{4-6}$ Doppler-free two-photon absorption ${ }^{7-11}$ and supersonic nozzle beam spectroscopy. ${ }^{12-14}$ Others, like hole burning or fluorescence line narrowing ${ }^{15,16}$ may be applied to solutions or glasses. All these methods expose a narrowed distribution of the transitions between the electronic ground state and some excited state of the molecule, and they may usually only be applied to low

\footnotetext{
a) This research was supported by a grant from USARO(D) (DAAG-29-80-C-0014) and in part by the NSF/MRL program under Grant No. DMR-7923647.

b) A research Fellowship of the "Deutsche Forschungsgemeinschaft" is gratefully acknowledged.
}

lying excited states. In order to obtain the relaxation parameters corresponding to a pair of excited states a method based on a linear optical effect requires one of these excited states to be initially populated. This is generally a difficult situation to achieve because of wide variations in the lifetimes of the states of interest.

Nonlinear coherent optical effects were used for spectroscopy shortly after tunable lasers became available. The resulting methods, typified by CARS, CSRS, and polarization labeling spectroscopy, are now well established and widely used. A microscopic theory of the nonlinear susceptibilities reveals that they contain several resonances not only associated with transitions from the ground state, but also with transitions between excited states. ${ }^{17-21}$ Additional interesting situations arise when the frequencies of the interacting fields are chosen in such a way that several resonances occur simultaneously. This not only leads to dramatic enhancement of the signal, but also to line narrowing under certain conditions. Furthermore, the resulting line shapes may contain the needed dynamical information for transitions between pairs of excited states. ${ }^{17,19,22}$

All previous applications and discussions of nonlinear line narrowing spectroscopy are based on $\chi^{(3)}$ the third order nonlinear susceptibility. Several beam configurations and frequency combinations have been proposed and demonstrated, namely, fully resonant CARS and CSRS and their predicted line narrowing, ${ }^{23-25}$ polarization spectroscopy, ${ }^{26}$ population grating, and phase conjugate configuration methods. ${ }^{27,28}$ The symmetry properties of $\chi^{(3)}$ make these methods widely applicable to crystals and glasses, even solutions, and usually no difficulties arise in obtaining phase matching, if required. There are, however, some disadvantages in using the third-order response. In CARS and CSRS two in-going and one out-going beam interact with a four level system. Therefore with two input frequencies the condition of being fully resonant can only be met if the vibrational frequencies are equal in the ground and excited states. Since this is usually not the case, always one resonance will be slightly detuned. In techniques 
which involve only a three level system (e.g., polarization spectroscopy), full resonance may be obtained, but the generated light will have the same frequency as one of the in-going beams. Therefore signal discrimination can be a limiting factor.

Up to now, effects based on the second order susceptibility $\chi^{(2)}$ have not been studied in the fully resonant configuration, and only a few experiments have been reported on resonance enhanced second harmonic generation $^{29,50}$ in organic systems. One reason why $\chi^{(2)}$ resonant responses have been neglected is that sum and difference frequency generation through dipolar interactions are restricted to noncentrosymmetric media and not applicable to isotropic systems. However, the needed anisotropy may always be induced by means of a static electric field of any sort, so this is not a fundamental restriction. The purpose of the present paper is, therefore, to study the spectroscopic properties of $\chi^{(2)}$ in the fully resonant configuration. It is shown that $\chi^{(2)}$ provides new information about the relaxation between pairs of excited states. Under certain circumstances $\chi^{(2)}$ also has line narrowing properties that could prove to be useful in condensed phase spectroscopy.

The second order susceptibilities discussed in this paper are amenable to study by a variety of techniques in a wide range of molecular systems. Some of the more obvious applications involve molecular crystals, mixed crystals, polymers, and surfaces. Noncentrosymmetric crystalline materials could be employed as host lattices for dipolar molecules whose spectra and dynamics are to be explored. Centrosymmetric host materials may also be used in sum and difference frequency generation experiments but a low frequency electric field is also necessary. The resonant parts of $\chi^{(2)}$ may be very large in such cases. These mixed crystal systems offer the opportunity to study moderately sharp optical and infrared transitions in the presence of relatively small inhomogeneous line broadening. Organic polymers and glasses which can be poled by electric fields could also be employed in the experiments. In these cases the line narrowing characteristics of $\chi^{(2)}$ spectroscopies could be more fully explored because the inhomogeneous distributions give rise to spectra much wider than the intramolecular decay widths. Molecules adsorbed on surfaces provide still another class of systems that are intrinsically noncentrosymmetric, in fact, resonance enhanced sum generation was recently shown for the case rhodamine dyes on fused silica. ${ }^{30}$

In the following we will discuss a molecular three level model for $\chi^{(2)}$ in the fully resonant condition. The next section is dedicated to the effect of electric. field parameters. This involves the solution of Maxwell's equations leading to formulas for phase matching conditions and build up of the signal. Time domain effects are not treated explicitly here. Section IV then deals with the predicted line shapes of the resonances under various experimental conditions. In Sec. $V$ the line narrowing properties of $\chi^{(2)}$ are presented and some examples for different types of inhomogeneous distributions are given. Some new types of line narrowing spectroscopy are suggested.

\section{MICROSCOPIC THEORY OF $\chi^{(2)}$ UNDER FULLY RESONANT CONDITIONS}

The quantity usually measured in nonlinear spectroscopy is the intensity of the light beam generated in the nonlinear medium through coupling of the in-going beams. The calculation of this intensity may be done in three steps. The first step involves the calculation of the nonlinear susceptibilities from the material constants of the medium. In the second step the in-going fields are coupled through the susceptibility to yield the nonlinear polarization. The latter, finally, acts as a source term in Maxwell's equation determining the build up of the signal beam. ${ }^{31}$

In this section we calculate $\chi^{(2)}$ in terms of quantum mechanical matrix elements of a molecular system. The connection between $P^{(2)}, \chi^{(2)}$, and the fields is, in general

$$
P^{(2)}(\omega)=\int_{-}^{\infty} d \omega^{\prime} \chi\left(\omega^{\prime}, \omega-\omega^{\prime}\right) E\left(\omega^{\prime}\right) E\left(\omega-\omega^{\prime}\right) .
$$

Here, $P(\omega)$ and $E(\omega)$ denote the Fourier transform:

$$
E(\omega)=\int_{-\infty}^{+\infty} \frac{d t}{2 \pi} E(t) \cdot e^{-i \omega t} .
$$

If the field is chosen as a sum of monochromatic waves, the integral equation (1) reduces to a simple product for each of the possible sum or difference frequency combinations:

$$
\mathrm{p}^{(2)}=\chi^{(2)}: \mathrm{EE} \text {. }
$$

Quantum mechanically the second order polarization is calculated as

$$
\mathbf{P}^{(2)}=\operatorname{Tr}\left(\rho^{(2)} \cdot \mu\right) .
$$

Here, $\mu$ is the dipole operator, and $\rho^{(2)}$ the second or der density matrix obtained from a perturbation expansion in powers of the fields. Therefore $\rho^{(2)}$ is quadratic in the electric fields. When the fields in the perturbation are chosen to be monochromatic, the quantum mechanical result can be directly compared with Eq. (3) yielding the microscopic expressions for $\chi^{(2)}$ as the coefficients of the various Fourier components of $P^{(2)}$ in Eq. (4). Since $\chi^{(2)}$ is a material constant, the choice of monochromatic waves is not a restriction. In practical applications, of course, the finite frequency distribution of the fields (caused, e.g. , through the finite duration of pulsed lasers), must be taken into account using Eq. (1).

The equation of motion for the density operator is given by the Liouville equation $(\hbar=1)$;

$$
\dot{\rho}=i[\rho, V]+(\dot{\rho})^{R} \text {. }
$$

All operators are in the interaction picture and the perturbation $V(t)$ is described by the interaction of a classical field $E(t)$ with the dipole $\mu(t)$ of the charged particles: $V(t)=-\mu(t) E(t) ; \mu_{\alpha \beta}(t)=\mu_{\beta \alpha}^{*}(t)=\mu_{\alpha \beta} \cdot \exp \left(i \omega_{\alpha \beta} t\right)$. The electric field is

$$
E(t)=\sum_{n=1,2}\left\{E_{n}^{*} \cdot \exp \left(-i \omega_{n} t\right)+E_{n} \exp \left(i \omega_{n} t\right)\right\} .
$$




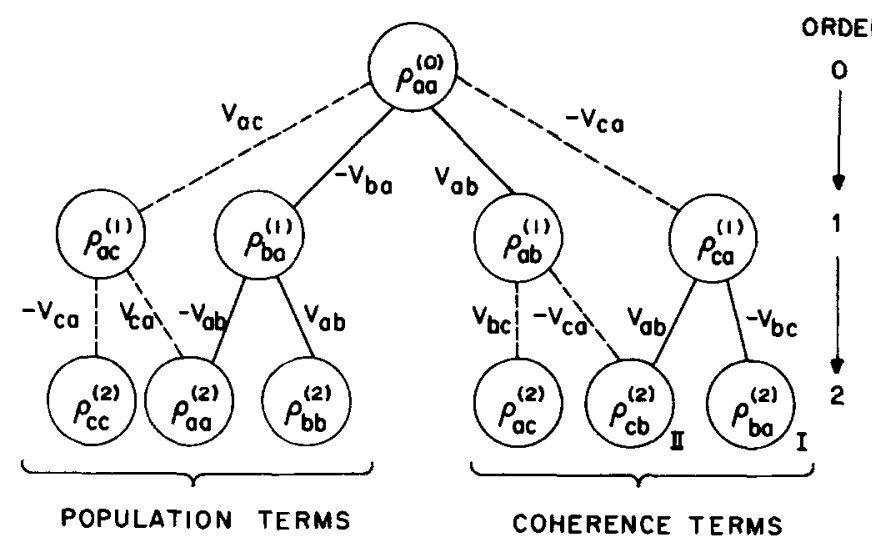

FIG. 1. Diagram illustrating the evolution of the resonant parts of the density operator to second order in the applied fields. The field components involved are represented by the broken $\left(\omega_{1}\right)$ and full $\left(\omega_{2}\right)$ correlation lines.

Since in second order a maximum of two fields can interact at a time, we may restrict ourselves to consider only two frequencies $\omega_{n}=\omega_{1}, \omega_{2}$. Both the positive and negative sign of the frequency are included in order to account properly for both counterrotating parts of the waves. The operator $(\dot{\rho})^{R}$ accounts for relaxation due to interactions with the baths. Its matrix elements are given in terms of the phenomenological (Markovian) damping parameters $\Gamma$ (coherence loss) and $\gamma$ (population loss):

$$
\begin{aligned}
& (\dot{\rho})_{\alpha \alpha}^{R}=-\gamma_{\alpha \alpha} \rho_{\alpha \alpha}+\sum_{\beta \neq \alpha} \gamma_{\beta \alpha} \rho_{B \beta}, \\
& (\dot{\rho})_{\alpha \beta}^{R}=-\Gamma_{\alpha \beta} \rho_{\alpha \beta} .
\end{aligned}
$$

The feeding terms $\gamma_{B \alpha} \rho_{B B}$ couple the equations of motion of the diagonal elements. They would, however, only contribute to the fully resonant second order terms if the starting populations were different from the steady state populations. Since we will assume all molecules to be in the ground state before the interaction with the fields $\left[\rho_{a a}^{(0)}(-\infty)=1\right]$, we can neglect all feeding terms. The solution to Eq. (5) then reads:

$\rho_{\alpha \beta}(t)=i \exp \left(-\Gamma_{\alpha \beta} t\right) \int_{-\infty}^{t} d t^{\prime} \exp \left(\Gamma_{\alpha \beta} t^{\prime}\right)\left[\rho\left(t^{\prime}\right), V\left(t^{\prime}\right)\right]_{\alpha \beta}$.

This integral equation can be solved iteratively after expanding $\rho$ in powers of the perturbation $V$. Before going to the results for second order it is of interest to trace the development of $\rho$ through the various orders using the diagram Fig. 1. This diagram, which may be easily extended to higher orders, is especially helpful in determining all important terms for the fully resonant case. It is based on the fact that a given matrix element $\rho_{\mu \nu}^{(n-1)}$, by virture of the commutator, may couple through $V_{\nu \sigma}$ to contribute to $\rho_{\mu \sigma}^{(n)}$, or with $-V_{\sigma \mu}$ to contribute to $\rho_{\sigma \nu}^{(n)}$. In the first case the bra part of the density operator is changed, while in the second case the $V$ operator with the negative sign changes the ket of $\rho$, so that our diagram is analogous to the double sided diagrams presented by Yee and Gustafson. ${ }^{32}$ Thus, starting from $\rho_{a a}^{(0)}=1$, in first order, all conjugate pairs of matrix elements of type $\rho_{a \nu}^{(1)}$ and $\rho_{v a}^{(1)}$ are generated. In the general case, all levels $\nu$ have to be considered, and each matrix element contains both frequencies $\omega_{1}$ and $\omega_{2}$ with positive and negative signs. Since we are interested in the resonant contributions, we may consider only two excited levels, namely, $b$ and $c$. This leads to matrix elements $\rho_{a c}, \rho_{b a}, \rho_{a b}$, and $\rho_{c a}$ in first order, which are resonant terms with frequencies chosen as $\omega_{2},-\omega_{1}, \omega_{1}$, and $-\omega_{2}$, respectively.

In second order, application of the same procedure leads to population terms and coherence terms. Only one of each complex conjugate pair of contributions to the population terms is shown in Fig. 1. Under fully resonant conditions these terms incorporate the same optical frequency component as in the first order, but with the opposite sign. Therefore, these zero frequency (or DC) terms do not contribute to the generation of the optical frequency polarization in second order. The nonlinear optical signal comes from the coherence terms depicted on the right-hand side of Fig. 1. Obviously two different types of matrix elements evolve, which we name type I and type II since they describe different physical processes. The type I matrix element describes coherence between the ground state and an excited state, such as $\rho_{a c}^{(2)}$ or $\rho_{b a}^{(2)}$. There is only one path to this matrix element, and both $V$ operators involved in this path have the same sign. The type $I I$ matrix element $\rho_{c b}^{(2)}$ describes coherence between two excited states and contains contributions from two paths, both with alternating signs of $V$. This will be important for the line narrowing capabilities of the type II process which will be discussed in Sec. V. The general solution for a matrix element of $\rho^{(2)}$ is

$$
\begin{aligned}
\rho_{2}^{(2)}= & -\sum_{\text {patts }} \exp \left(-\Gamma_{2}\right) \int_{-\infty}^{t} d t^{\prime} V_{1-2}\left(t^{\prime}\right) \\
& \times \exp \left[\left(\Gamma_{2}-\Gamma_{1}\right) t^{\prime}\right] \int_{-\infty}^{t^{\prime \prime}} d t^{\prime \prime} V_{0 \rightarrow 1}\left(t^{\prime \prime}\right) \exp \left(\Gamma_{1} t^{\prime \prime}\right) \cdot(-1)^{n} .
\end{aligned}
$$

The dummy indices 1 and 2 must be replaced by the index pairs of the first and second order matrix element involved in the path, and $0-1$ or $1-2$ denote the indices of the corresponding $V$ operators. Finally, $n$ is the total number of $V$ operators occurring with negative sign in the path. The result of integration is

$$
\begin{aligned}
\rho_{2}^{(2)}= & -\sum_{\text {path }} \sum_{\alpha, \beta} \frac{\left(E_{\beta} \mu_{12}\right)\left(E_{\alpha} \mu_{01}\right)(-1)^{n}}{\left[i\left(\omega_{01}+\omega_{\alpha}\right)+\Gamma_{1}\right]\left[i\left(\omega_{01}+\omega_{12}+\omega_{\alpha}+\omega_{\beta}\right)+\Gamma_{2}\right]} \\
& \times \exp \left[i\left(\omega_{01}+\omega_{12}+\omega_{\alpha}+\omega_{\beta}\right) t\right] .
\end{aligned}
$$

The summation over the field components $\alpha$ and $\beta$ yields 16 contributions from each path, but the constraint of being fully resonant will allow only one or two combinations to occur.

The optical polarization is obtained from the trace of $\rho \mu$ :

$$
p^{(2)}=\rho_{b a}^{(2)} \cdot \mu_{a b}+\rho_{c b}^{(2)} \cdot \mu_{b c}+\rho_{a c}^{(2)} \mu_{c a}+c . c .
$$

The following susceptibilities for sum and difference frequency generation under fully resonant conditions are then readily found: 


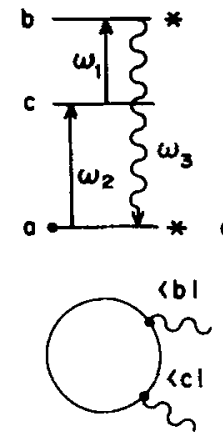

$|0\rangle\langle 0|$
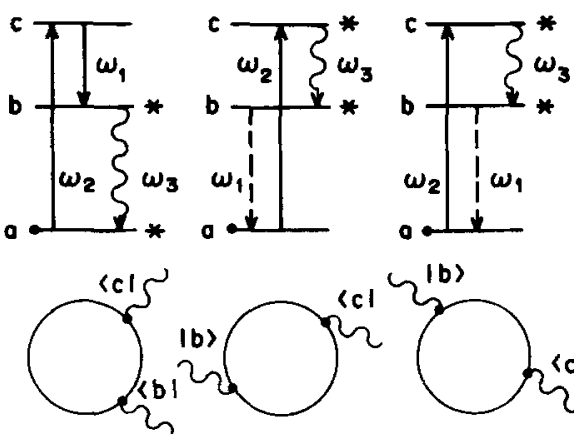

$|a\rangle\langle a|$

(b)

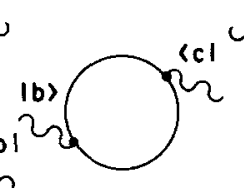

$|a\rangle\langle 0|$

(c)

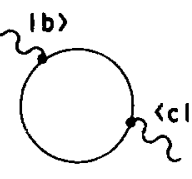

$|a\rangle\langle a|$
FIG. 2. Diagrammatic representation of the fully resonant second order susceptibilities. Time ordering is from left to right in each diagram. Upwards arrows indicate positive frequency components (photon annihilation), downward negative frequency (photon creation). Full and broken lines refer to evolution of the bra and ket parts, respectively, of the density operator. Asterisk mark the levels between which coherence is induced. The graphs (a) and (b) refer to $\rho_{a b}$, while (c) and (d) contribute to $\rho_{b c}$. The graphs for the complex conjugates $\rho_{b a}$ and $\rho_{c b}$ are found by reversing all arrow directions, changing full into broken lines, and vice versa. Below each diagram the corresponding graph of Yee and Gustafson (Ref. 32) which reduces to the diagram in the fully resonant cage is shown. Note that in case (a) the levels $b$ and $c$ have been reversed in order to maintain the strong similarity of the equations describing type I processes.

$$
\begin{aligned}
& \chi_{\mathrm{I}}^{(2)}\left(-\omega_{s} ; \omega_{1}, \omega_{2}\right)=\frac{\mu_{a b}^{(s)} \mu_{b c}^{(1)} \mu_{c a}^{(2)}}{\left[\omega_{c a}-\omega_{2}+i \Gamma_{c a}\right]\left[\omega_{b a}-\omega_{s}+i \Gamma_{a b}\right]}, \\
& \chi_{\mathrm{I}}^{(2)}\left(-\omega_{D} ;-\omega_{1}, \omega_{2}\right)=\frac{\mu_{a b}^{(D)} \mu_{b c}^{(1)} \mu_{c a}^{(2)}}{\left[\omega_{c a}-\omega_{2}+i \Gamma_{c a}\right]\left[\omega_{b a}-\omega_{b}+i \Gamma_{a b}\right]},
\end{aligned}
$$

$$
\chi_{I 1}^{(2)}\left(-\omega_{D} ; \omega_{2},-\omega_{1}\right)=\frac{\mu_{b c}^{(D)} \mu_{a b}^{(1)} \mu_{a d}^{(2)}}{\left[\omega_{b a}-\omega_{1}+i \Gamma_{a b}\right]\left[\omega_{c b}-\omega_{D}-i \Gamma_{b c}\right]},
$$

$$
\chi_{\mathrm{II}}^{(2)}\left(-\omega_{D} ;-\omega_{1}, \omega_{2}\right)=\frac{-\mu_{b c}^{(D)} \mu_{c b}^{(1)} \mu_{c a}^{(2)}}{\left[\omega_{c a}-\omega_{2}-i \Gamma_{c a}\right]\left[\omega_{c b}-\omega_{D}-i \Gamma_{b c}\right]} .
$$

Here, $\omega_{s}=\omega_{1}+\omega_{2}$ and $\omega_{D}=\omega_{2}-\omega_{1}$ are the sum and difference frequencies with $\omega_{2} \geq \omega_{1}$ in the latter case. The field components to be taken with the various dipole moments are indicated by the superscripts. There are two contributions from the type I matrix element, one each for sum and difference frequency generation. In the type II process only difference frequency generation is possible, but again two contributions arise.

The physical meaning of Eqs. (12a)-(12d) is best explained with the diagrams in Fig. 2. In Fig. 2, the diagrams a-d correspond to Eqs. (12a)-(12d) having the same letter designation. Although the type I process may produce either sum or difference frequency polarization, the mechanism of the physical processes involved are quite similar. This is already obvious from the formulas for $\chi^{(2)}$, since Eqs. (12a) and (12b) only differ by the replacement of $\omega_{b}$ by $\omega_{s}$. As seen in the diagram, the frequency $\omega_{2}$ first couples the level $c$ to the ground state, while in the second step $\omega_{1}$ couples levels $c$ and $b$, thus finally introducing coherence between the level pair $a b$. The difference is only in the relative order of levels $b$ and $c$, leading to difference frequency generation if $b$ is below $c$, and to sum frequency generation if $b$ is above $c$. These processes differ quantum mechanically in that an $\omega_{1}$ photon is absorbed in (a) but emitted in (b). In the case of sum frequency generation, the generated wave will be in resonance with the transition from the ground state to a higher excited state of the molecule. The reabsorption of this wave is discussed in Sec. III.

The type II processes in the diagrams (c) and (d) obviously contribute to the same physical process, since they generate coherence between the same pair of levels $b$ and $c$ and only differ in the time ordering of the interactions. As long as monochromatic waves are considered, these two orderings cannot be distinguished.

The problem of reabsorption of the generated light encountered in type I processes will not occur in the type II process if the separation between levels $b$ and $c$ is smaller than the energy corresponding to the $a b-$ sorption edge. The generated wave at $\omega_{b}$ will then be well separated in frequency from $\omega_{1}, \omega_{2}$ and the fluorescence light $(b \rightarrow a$ or $c \rightarrow a)$, and it will travel in a medium which is nearly transparent for this frequency.

The type II process is especially interesting since the corresponding susceptibilities [Eqs. (12c) and (12d)] explicitly contain $\Gamma_{c b}$ as a linewidth parameter in a resonance. Thus it offers the possibility to measure this coherence decay parameter for a pair of excited levels, which are initially not populated. This is not possible in the type I process which only depends on the conventional spectroscopic damping parameters $\Gamma_{a b}$ and $\Gamma_{a c}$. In the type II process the levels may be vibrational levels of various electronic states, so that the decay of a large number of combinations of level pairs should be accessible. In order to facilitate the discussion in subsequent sections for formulas (12a)-(12d) can be contracted to a single formula for each type of process, leading to:

$$
\begin{aligned}
\chi_{\mathrm{I}}^{(2)}\left(-\omega_{3}\right)= & \frac{\mu_{a b}^{(3)} \mu_{b c}^{(1)} \mu_{c a}^{(2)}}{\left[\omega_{c a}-\omega_{2}+i \Gamma_{c a}\right]\left[\omega_{b a}-\omega_{3}+i \Gamma_{a b}\right]}, \\
\chi_{I I}^{(2)}\left(-\omega_{3}\right)= & \frac{\mu_{c b}^{(2)} \mu_{a b}^{(1)} \mu_{b c}^{(3)}}{\left[\omega_{b a}-\omega_{1}+i \Gamma_{a b}\right]\left[\omega_{c a}-\omega_{2}-i \Gamma_{a c}\right]} \\
& \times\left\{1+i \frac{\Gamma_{c b}-\Gamma_{a c}-\Gamma_{a b}}{\left[\omega_{c b}-\omega_{3}-i \Gamma_{c b}\right]}\right\} .
\end{aligned}
$$

For type I processes, $\omega_{3}$ may be either $\omega_{s}$ or $\omega_{D}$, while only $\omega_{D}$ is allowed in type II. Equation (13b) is obtained as the sum of Eqs. (12c) and (12d). In this form it exhibits an interesting feature of the extra $\omega_{c b}$ resonance. We describe the coherence decay parameters $\Gamma_{\alpha \beta}$ in the usual way as

$$
\Gamma_{\alpha \beta}=\frac{1}{2}\left(\Gamma_{\alpha}+\Gamma_{\beta}\right)+\Gamma_{\alpha B}^{\prime},
$$

with $\Gamma_{\alpha}$ and $\Gamma_{\beta}$ being the inverse total lifetimes of levels $\alpha$ and $\beta$ and $\Gamma_{\alpha \beta}^{\prime}$ the pure dephasing parameter. The inverse lifetime of the ground state is assumed to be zero, the population lifetimes cancel out in the numerator of 
the $\omega_{c b}$ resonance, and the resonance becomes proportional to $\Gamma_{c b}^{\prime}-\Gamma_{a c}^{\prime}-\Gamma_{a b}^{\prime}$. If the pure dephasing vanishes, as might occur at very low temperatures, the resonance of $w_{c b}$ will not occur. In this case the $\omega_{c b}$ resonance is predicted to be temperature induced, giving rise to a dephasing induced coherent emission (DICE) effect in second order having the same physical origin as that discussed earlier ${ }^{19,20,22}$ for third-order processes.

\section{OPTICS}

The intensity of the coherent signal generated by the nonlinear polarization along the direction of the ingoing beams is obtained from Maxwell's equations. For this purpose we have to consider the space dependence for all waves:

$$
E_{j}(\omega, r)=E_{j}(\omega) \cdot \exp (i \mathrm{~K} \cdot \mathbf{r}) ; j=1,2,3 .
$$

We may choose the wave vectors $K$ to be complex to account for the loss due to absorption in the medium:

$$
\mathrm{K}_{j}=\mathbf{e}_{j}\left(k_{j}+i a_{j}\right) \text {. }
$$

In this expression $e_{j}$ is the unit vector perpendicular to the traveling wavefront, $k_{j}=\eta \omega_{j} / 2 \pi c$ is the wave vector in the medium and $a_{j}$ half the linear absorption coefficient of the medium at $\omega_{j}$. In the slowly varying envelope approximation $^{31}$ the amplitude of the generated wave is

$A_{3}\left(\omega_{3}, L\right)=\frac{2 \pi i \omega_{3}}{c n_{3}} \cdot \int_{0}^{L} d z P^{(2)}\left(\omega_{3}, z\right) \exp \left(-i K_{3} z\right)$.

In the general case $P^{(2)}(\omega, z)$ is given by Eq. (1) including the space dependent factors for the fields according to Eq. (15). Therefore, in general, the integrals over $d z$ and $d \omega^{\prime}$ cannot be separated, unless the dispersion of the wave vectors can be neglected. We will assume the frequency distributions around $\omega_{1}, \omega_{2}$, and $\omega_{3}$ to be small enough to justify such a neglect, leading to the result:

$$
\begin{aligned}
I_{3}= & \left|A_{3}\right|^{2} \cdot \exp \left(-2 a_{3} L\right)=\left(\frac{2 \pi \omega_{3}}{c n_{3}}\right)^{2}\left|P_{Z=0}^{(2)}\left(\omega_{3}\right)\right|^{2} \cdot G(\Delta k) \\
& \times \exp \left(-2 a_{3} L\right) .
\end{aligned}
$$

The phase mismatch factor is given by

$$
G(\Delta k)=\frac{1+\exp [-2 a L]-2 \exp [-a L] \cdot \cos (\Delta k L)}{(\Delta k)^{2}+a^{2}}
$$

with

$$
a=a_{1}+a_{2}-a_{3}
$$

and

$$
\Delta k=k_{3}-\left(k_{1}^{2}+k_{2}^{2} \pm 2 k_{1} k_{2} \sin \theta_{12}\right)^{1 / 2} .
$$

$\Delta k$ is the scalar phase mismatch for sum frequency generation (+ sign) and difference frequency generation (-sign), respectively. $\theta_{12}$ is the angle between the two in-going beams.

It is obvious, that in a system having a refractive index that monotonically increases with $\omega, \Delta k$ cannot be made equal to zero by varying $\theta_{12}$.

\section{LINE SHAPES}

In a nonlinear spectroscopic experiment the frequencies of the in-going beams are varied and the change of signal intensity observed. When the phase mismatch factor is effectively constant during this scan, the line shapes obtained are entirely determined by $\left|P^{(2)}\right|^{2}$ or, in the case of monochromatic waves, by $\left|\chi^{(2)}\right|^{2}$. Since there are two in-going beams $\omega_{1}$ and $\omega_{2}$, every line in the $\left(\omega_{1}, \omega_{2}\right)$ plane could define a possible scan associated with a line shape. The situations that may be most easily realized are scan modes in which one of the frequencies involved remains fixed. Three scan modes of this type are possible in a $\chi^{(2)}$ experiment. In two of them one of the in-going beams is fixed while the other is scanned. In the third case, in order to fix the generated frequency $\omega_{3}$, the ingoing beams must be scanned maintaining constant $\left(\omega_{1}-\omega_{2}\right)$ for difference frequency generation, or constant $\left(\omega_{1}+\omega_{2}\right)$ for sum frequency generation. Line shapes predicted for these scan modes under fully resonant conditions are discussed below in relation to the structural information that may be obtained in each particular case. For simplicity we will assume the in-going beams to be monochromatic, so that the lineshapes are entirely determined by the $\chi^{(2)}$ given in Eqs. (13a) and (13b) for the type I and type II process, respectively. It is first assumed that no inhomogeneous broadening is present.

The three scan modes yield only three different types of line shapes:

type A: $\quad I_{3} \propto\left|\frac{M}{\left(\omega_{x}-\omega\right)+i \Gamma_{x}} \pm \chi\right|^{2}$,

type B: $\quad I_{3} \propto\left|\frac{1}{\left[\left(\omega_{x}-\omega\right)+i \Gamma_{x}\right]\left[\left(\omega_{y}-\omega\right)+i \Gamma_{y}\right]} \pm \chi\right|^{2}$,

type C: $\quad I_{3} \propto\left|\frac{M}{\left[\left(\omega_{x}-\omega\right)+i \Gamma_{x}\right]}\left\{1+i \frac{\Gamma_{c b}-\Gamma_{a c}-\Gamma_{a b}}{\left(\omega_{y}-\omega\right)+i \Gamma_{y}}\right\} \pm \chi\right|^{2}$.

In general, $M$ is complex, referring to the resonance denominator containing the fixed frequency in the form $\left(\omega+i \Gamma_{f}\right)^{-1}$, where $\Gamma_{f}$ describes the damping of the coherence introduced by the fixed beam. Type $A$ exhibits one resonance at $\omega_{x}$, while $\mathrm{B}$ and $\mathrm{C}$ each show a further resonance at $\omega_{y}$. The corresponding linewidth parame-

\begin{tabular}{|c|c|c|c|c|c|c|}
\hline \multicolumn{2}{|c|}{$\begin{array}{l}\text { Fixed } \\
\omega \\
\end{array}$} & \multirow{2}{*}{$\begin{array}{l}\begin{array}{l}\text { Line- } \\
\text { type }\end{array} \\
\text { B }\end{array}$} & \multirow{2}{*}{$\frac{\omega_{x}}{\omega_{\infty}}$} & \multirow{2}{*}{$\frac{\omega_{y}}{\omega_{D a} \mp \omega_{1}}$} & \multirow{2}{*}{$\frac{\Gamma_{x}}{\Gamma_{a c}}$} & \multirow{2}{*}{$\frac{\Gamma_{y}}{\Gamma_{a b}}$} \\
\hline & $\omega_{1}$ & & & & & \\
\hline \multirow[t]{3}{*}{ I } & $\omega_{2}$ & A & $\omega_{b c}-\omega_{c}$ & & $\Gamma_{a b}$ & $\cdots$ \\
\hline & $\omega_{3}$ & A & $\omega_{\infty}$ & $\cdots$ & $\Gamma_{\infty}$ & $\cdots$ \\
\hline & $\omega_{1}$ & $\mathrm{C}$ & $\omega_{\alpha}$ & $\omega_{\infty}+\omega_{1}$ & $-\Gamma_{a c}$ & $-\Gamma_{\infty}$ \\
\hline \multirow[t]{2}{*}{ II } & $\omega_{2}$ & $\mathrm{C}$ & $\omega_{b a}$ & $\omega_{c b}-\omega_{2}$ & $\Gamma_{a b}$ & $-\Gamma_{\infty}$ \\
\hline & $\omega_{3}$ & B & $\omega_{b a}$ & $\omega_{a}-\omega_{3}$ & $\Gamma_{a b}$ & $-\Gamma_{a c}$ \\
\hline
\end{tabular}
ters are named $\Gamma_{x}$ and $\Gamma_{y}$, respectively. The actual parameter to be substituted for these variables in the three scan modes for type I and type II processes are collected in Table I. We also have included a non-

TABLE I. Resonance parameter to be used in the line shape calculations. 

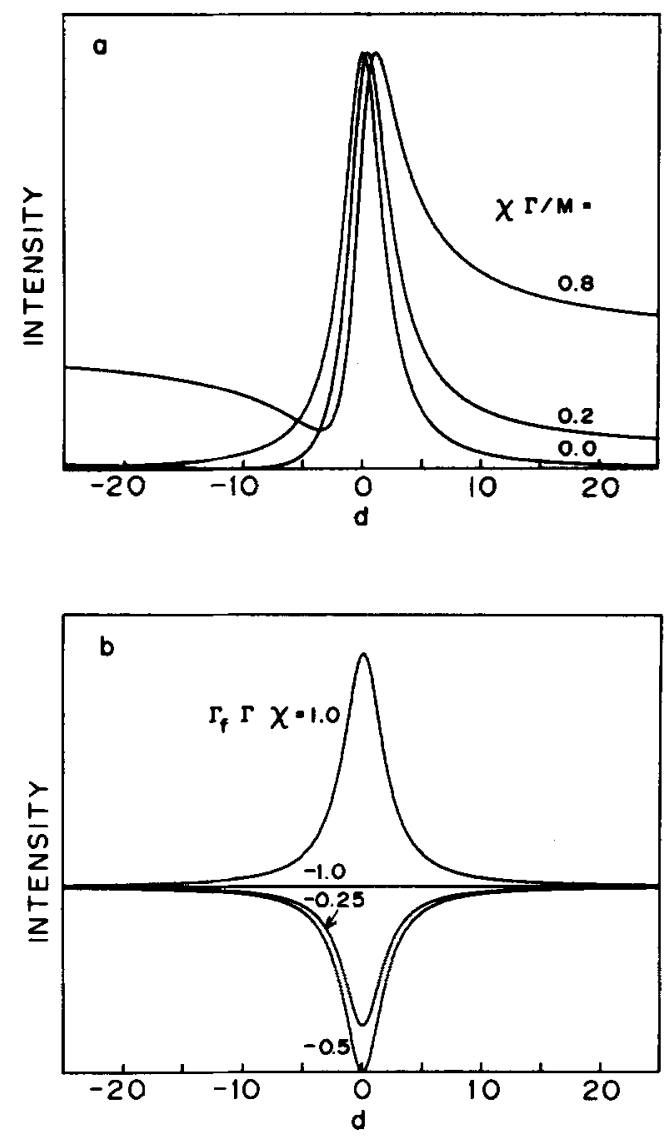

FIG. 3. Line shapes of type A resonances for various values of the nonresonant background. (a) Fixed frequency from resonance, (b) fixed frequency on resonance, $\Gamma=2$ in all lines. For details see the text.

resonant background factor $\chi=\chi_{\mathrm{XR}} /\left|\mu_{\mathrm{ab}} \mu_{\mathrm{bc}} \mu_{c a}\right|$, which may interfere with the resonances. The line shape type A is predicted to occur when either $\omega_{2}$ or $\omega_{3}$ [in Eq. (13a) ] is fixed. When the nonresonant background is negligible the line will be a Lorentzian having width $2 \Gamma_{b a}$ or $2 \Gamma_{c a}$. On the other hand, when the nonresonant background is significant the line shape depends on the amount of detuning of the fixed frequency and on whether the + or - sign is chosen in Eq. (22). The two line shapes shown in Fig. 3 correspond to large (a) and zero (b) detuning of the fixed frequency from resonance. For large detuning the line is expected to be asymmetric and the peak position and peak to minimum separation can be used to determine some of the resonant parameters in relation to the nonresonant background as indicated in Fig. $3(\mathrm{a}) .{ }^{33}$

The sign in Egs. (22) -(24) must be chosen as the sign of $\mu_{a b} \mu_{b c} \mu_{c a}$. In this case the line shape and the peak to background ratio are sensitively dependent on this choice. The different signs for the transition dipole factor are related to the details of the vibrational-electronic transitions involved and in the simplest cases can be deduced from the signs of the associated FranckCondon factors. One possible situation could have each of $\mu_{c b}, \mu_{c a}$, and $\mu_{b c}$ corresponding to transitions between zero-point levels of different electronic states.
In this case the + sign is indicated. On the other hand, if the vibrational quantum number is changed during one or more of the transitions, the sign choice would depend on the details of the equilibrium nuclear configurations in each of the three states.

The line shape B occurs in type I and type II processes where the frequency connecting the two excited levels $b$ and $c$ is fixed. In the type I process this was called $\omega_{1}$, while in the type II process it is the signal frequency. This line shape is the product of two Lorentzians resulting in two maxima in the detuned case. By the proper choice of the fixed frequency, however, $\omega_{x}$ and $\omega_{y}$ can always be brought into coincidence, and it is just this situation that leads to the fully resonant case we are most interested in for spectroscopic applications. With the fixed frequency on resonance the signal intensity has the form

$$
\frac{1 \pm 2 \chi\left(\vec{\omega}^{2}-\Gamma_{x} \Gamma_{y}\right)}{\left(\omega^{2}+\Gamma_{x}^{2}\right)\left(\vec{\omega}^{2}+\Gamma_{y}^{2}\right)}+\chi^{2}
$$

When $\chi$ is relatively small the lines appear Lorentzian with widths ranging from the smallest of $\Gamma_{x}$ and $\Gamma_{y}$ to $0.64 \Gamma_{x}$ when $\Gamma_{x}=\Gamma_{y}$ [see Fig. 4(a)]. When $\chi$ is significant the spectra are expected to show a number of interesting features as shown in Fig. $4(b)$. The line may show a sharp dip which reaches the nonresonant back-
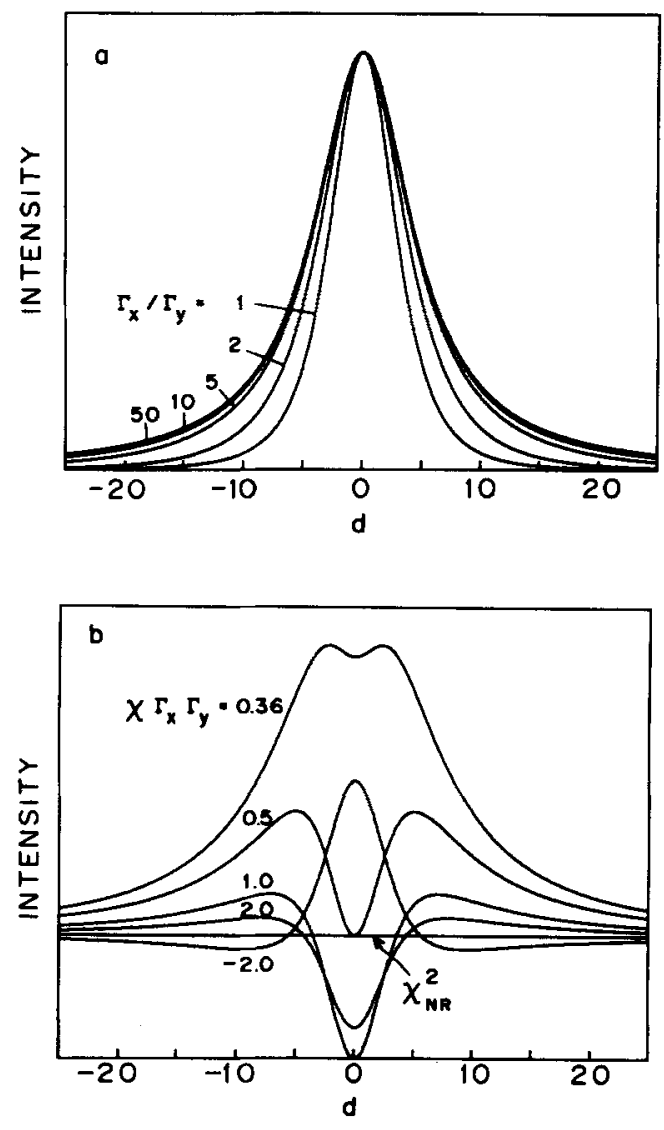

FIG. 4. Line shapes for type B resonances: (a) With nonresonant background $0, \Gamma_{x}$ fixed at 5 , and $\Gamma_{y}$ varied. All lines are normalized to the same peak value. (b) Effect of the nonresonant background. The nonresonant background is fixed and the amplitude varied. Both $\Gamma$ parameters are set to 5 . 

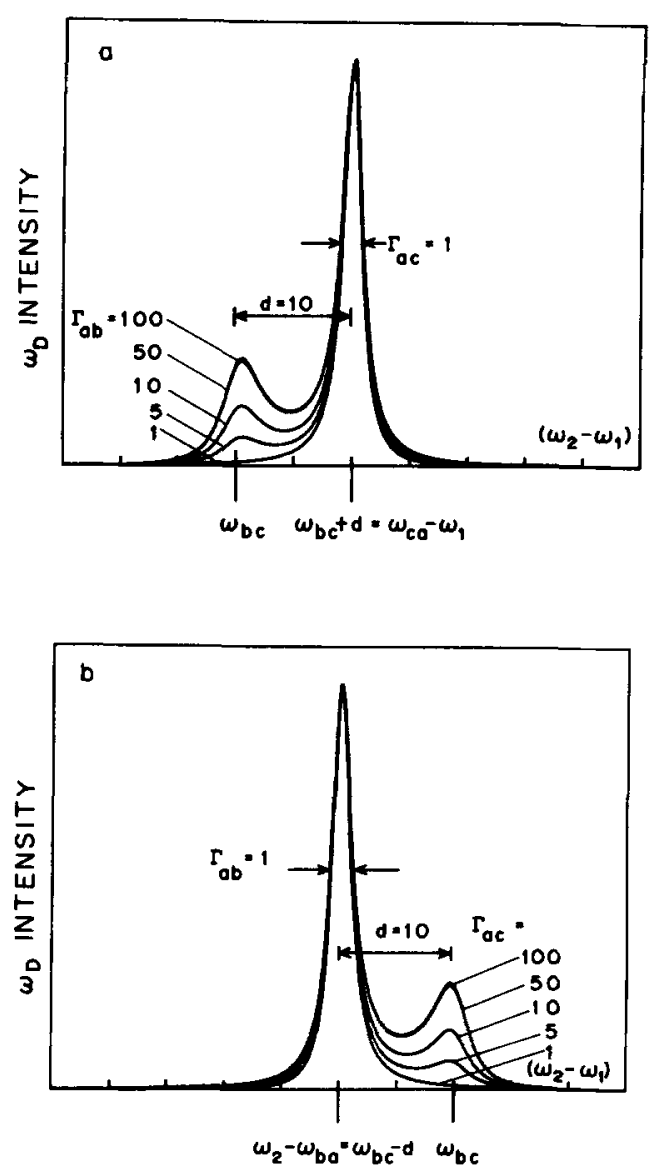

FIG. 5. Simulation of the DICE extra resonance in the type II susceptibility. (a) $\omega_{1}$ fixed, $\Gamma_{a b}$ varied, $\Gamma_{a c}=1, \Gamma_{b c}=2$; (b) $\omega_{2}$ fixed, $\Gamma_{a c}$ varied, $\Gamma_{a b}=1, \Gamma_{b c}=2$. The fixed frequency is detuned by 10 below the corresponding resonance.

ground level when $2 \chi \Gamma_{x} \Gamma_{y}=1$. The width of this dip can be smaller than the $\Gamma$ parameters. Other interesting cases are summarized in Fig. 4.

The line types discussed so far have in common that they allow the determination of $\Gamma_{a b}$ and $\Gamma_{a c}$, but not the relaxation parameter $\Gamma_{b c}$ between the two excited states. This measurement is possible with the type II C process when one of the in-going beams has a fixed frequency. In the general case these line shapes may become very complex, since they contain two resonances, three $\Gamma$ parameters, and a nonresonant background. Some special cases of interest are simulated in Fig. 5 . The resonant signal shape is very sensitive to the relative magnitudes of the $\Gamma$ parameters. Neglecting $\chi_{\mathrm{NR}}$ for the moment we will see that the simplest case arises if $\Gamma_{a b}+\Gamma_{a c}=\Gamma_{b c}$, since the second resonance then vanishes and the resulting line is a simple Lorentzian having the linewidth $\Gamma_{x}=\Gamma_{a b}$ or $\Gamma_{a c}$. As mentioned in Sec. II this case could apply at very low temperatures if the pure dephasing were vanishingly small. The effect of increasing $\Gamma=\Gamma_{a b}+\Gamma_{a c}-\Gamma_{b c}$ is most evident in a configuration where $\omega_{1}$ or $\omega_{2}$ is detuned from exact resonance. For example, if $\omega_{1}$ is detuned by $d$ below $\omega_{b a}$ and $\omega_{2}$ is scanned the results of Fig. 5(a) are obtained. A single line having the width $\Gamma_{a c}$ is observed when $\Gamma=0$, but at finite $\Gamma$ an extra resonance appears at $\omega_{2}-\omega_{1}=\omega_{b c}$ corresponding to the interexcited state transition. When $\omega_{2}$ is fixed at $d$ below $\omega_{c a}$ the extra resonance now appears on the high difference frequency side of the shifted one photon resonance as shown in Fig. 5(b).

\section{LINE NARROWING}

The observed coherent light signal is generated by the polarization, so if the medium is inhomogeneous the polarization is the sum of the various oscillating dipoles that are induced in the sample by the driving fields. The characteristic frequency width of the inhomogeneous distribution may be the main factor determining the widths of the nonlinear spectra, on the other hand, the spectra may be line narrowed.

The line narrowing properties of $\chi^{(2)}$ and $\chi^{(3)}$ processes under fully resonant conditions are dependent on the result of averaging the susceptibility over the inhomogeneous distribution. This averaging requires the definition of an appropriate model for such an inhomogeneous distribution of energies for both levels $b$ and $c$ with respect to the ground state $a$. A particular molecule will then be characterized by two parameters $x$ and $y$, giving the shift of the transition frequencies $\omega_{b a}$ and $\omega_{c a}$ from their mean values:

$$
\begin{aligned}
& x=\omega_{b a}-\omega_{b a}^{0}, \\
& y=\omega_{c a}-\omega_{c a}^{0} .
\end{aligned}
$$

Thus, the susceptibility of each molecule will depend on both $x$ and $y$ and the susceptibility of the whole ensemble will be

$$
\left\langle\chi^{(2)}\right\rangle=\iint d x d y \chi^{(2)}(x, y) \cdot G(x, y),
$$

where $G(x, y)$ is the joint distribution function. Obviously the distribution function $G$ must contain some correlation between the variables $x$ and $y$. Otherwise, the choice of a given $x$ (e.g., by resonance with a laser beam) will always project out the same distribution of $y$, and line narrowing cannot occur.

A simple model for the distribution function that has all the features required is the bivariate probability density:

$$
G(x, y)=g\left(\xi, \sigma_{1}\right) \cdot g\left(\eta, \sigma_{2}\right),
$$

$G(x, y)$ is schematically shown in Fig. 6. Here $(\xi, \eta)$ is an orthogonal axis system tuned by an angle $\phi$ with respect to $(x, y)$, and the $g$ are one dimensional distribution functions having widths $\sigma_{1}$ and $\sigma_{2}$. A measure of the correlation between the two variables $x$ and $y$ is their correlation coefficient $q$ which is given by

$$
q=\langle x y\rangle /(\langle x x\rangle\langle y y\rangle)^{1 / 2} .
$$

From this definition it follows that $1>q>-1$. The case $q=1$ is fully correlated, while $q=-1$ is anticorrelated. A correlation coefficient for a Lorentzian distribution having the same limiting characteristics as the Gaussian can be defined. ${ }^{34}$ If one of the two widths $\sigma_{1}$ and $\sigma_{2}$ becomes much smaller than the other, the correlation becomes more pronounced. In the case of vanishing $\sigma_{2}$, we find $q= \pm 1$ and each $x$ value allows only one $y$ value. 


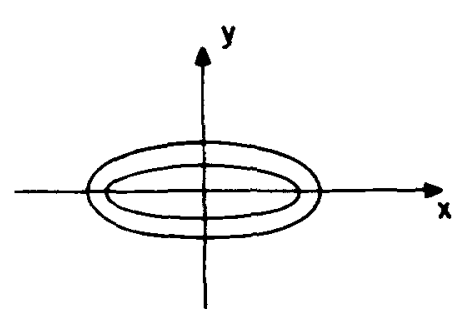

(a)
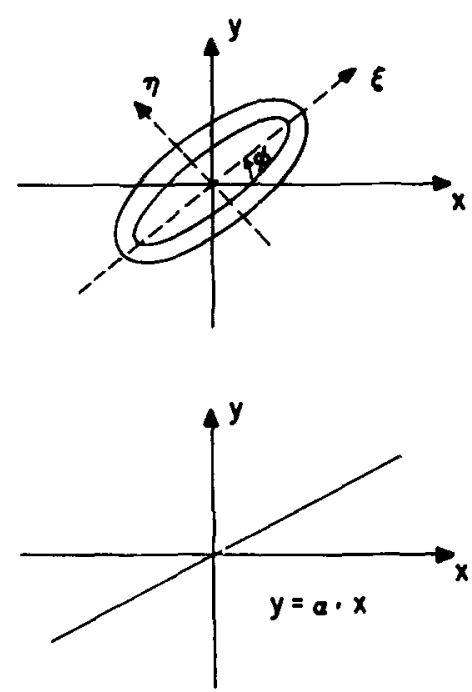

(c)

FIG. 6. Different models for inhomogeneous distributions of three level systems: (a) without correlation, (b) intermediate correlation, (c) strict correlation limit $|q|=1$.

The distribution function then reduces to the form:

$$
G(x, y)=g\left(x, \sigma_{1}\right) \cdot \delta(y-\alpha x) .
$$

An example of such a distribution occurs in the Doppler broadening of optical transitions in the gas phase $(q=1)$. The parameter $\alpha=\tan \phi$ is then equal to the ratio of the inhomogeneous widths of the two excited states $c$ and $b$. In the solid state the inhomogeneous distribution is often considered to be a result of different sites having different transition energies but the same sets of dynamical parameters. The treatment of intermediate $q$ values needed in this case is possible but the results are cumbersome. In the case of Gaussian distributions a numerical integration is required. Therefore, only the case $q= \pm 1$ is dealt with in detail while our results for other cases are summarized at the end of this section.

With respect to the nature of the distribution function $g(x, \sigma)$ the most natural choice would be a Gaussian, especially in the case that the inhomogeneous distribution were the result of a large number of statistically independent perturbations:

$$
g_{G}(x, \sigma)=\frac{1}{\sigma \sqrt{2 \pi}} \exp \left\{-x^{2} / 2 \sigma^{2}\right\} .
$$

If the inhomogeneous distribution is much wider than the set of homogeneous linewidths, the wings of the distribution function become less important, and the distribution may be approximated by a Lorentzian:

$$
g_{L}(x, \sigma)=\frac{\sigma}{\pi} \cdot \frac{1}{x^{2}+\sigma^{2}} \text {. }
$$

The solutions for Lorentzian distributions lead to expressions which can be readily interpreted, therefore they will be derived here. The numerical line shape simulations with both Gaussian and Lorentzian distributions indicate that the narrowed lines are essentially the same for both distribution functions (see Fig. 8). This justifies the choice of the Lorentzian a posteriori, and suggests that the particular form of the distribution function encountered in practice may not significantly influence the results.

In the limit $q \approx \pm 1$ and with Lorentzian distributions, the ensemble averaged susceptibility takes the form:

$$
\left\langle\chi^{(2)}\right\rangle=\int d x \chi^{(2)}(x) \frac{\sigma / \pi}{x^{2}+\sigma^{2}},
$$

$\chi^{(2)}(x)$ is obtained from Eq. (13a) or (13b) for type I or type II processes by substituting $\omega_{c a}=\omega_{c a}^{0}+\alpha x, \omega_{b a}$ $=\omega_{b a}^{0}+x$, and $\omega_{c b}=\omega_{c b}^{0}+(\alpha-1) x$. All the resulting integrals may be solved by means of the residue theorem. The appropriate integration paths and important poles are indicated in Fig. 7 for the various conditions that can occur.

Type I process: The integrand in the type I process
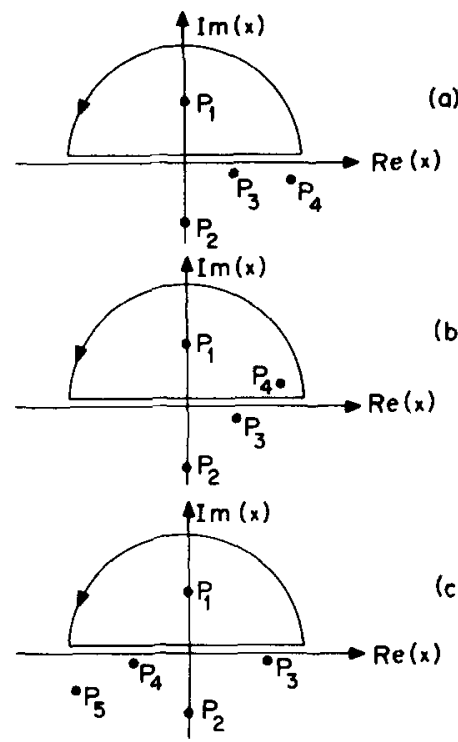

(a)
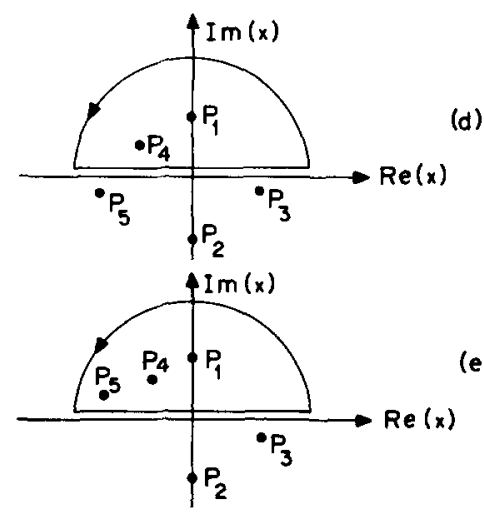

FIG. 7. Integration contours and poles in the complex plane for the line narrowing calculation with a Lorentzian inhomogeneous distribution. (a) type I, $\alpha>0$; (b) type I, $\alpha<0$; (c) type II, $\alpha<0$, (d) type II, $0<\alpha<1$; (e) type II, $\alpha>1$. 
has two poles from the distribution function and two poles from the susceptibility: $p_{1}=i \sigma ; p_{2}=-i \sigma ; p_{3}=\omega_{3}$ $-\omega_{b a}^{0}-i \Gamma_{a b} ;$ and $p_{4}=\left[\omega_{2}-\omega_{c a}^{0}-i \Gamma_{c a}\right] / \alpha$. Only those poles on one-half of the complex plane need be considered. If the correlation is positive, this is $\alpha>0$, the pole $P_{1}$ is the only one above the real axis, and $\left\langle\chi^{(2)}\right\rangle$ is given by the residue at this pole (case 1 , type I):

$\left\langle\chi_{I}^{(2)}\right\rangle_{1}=\frac{\mu_{a b}^{(3)} \mu_{b c}^{(1)} \mu_{c a}^{(2)}}{\left[\omega_{c a}^{0}-\omega_{2}+i\left(\Gamma_{c a}+\alpha \sigma\right)\right]\left[\omega_{b a}^{0}-\omega_{3}+i\left(\Gamma_{a b}+\sigma\right)\right]}$

Obviously the inhomogeneous width adds to both linewidths and no line narrowing occurs. This is a general property of the residues at the poles of the distribution function. In the case $\alpha<0$ the pole $p_{4}$ shifts above the real axis [see Fig. $7(b)$ ] and its residue has to be added to that of $p_{1}$ in this situation:

$$
\begin{aligned}
& \left\langle\chi_{\mathrm{i}}^{(2)}(\alpha<0)\right\rangle=\left\langle\chi_{\mathrm{I}}^{(2)}\right\rangle_{1}+\left\langle\chi_{\mathrm{I}}^{(2)}\right\rangle_{2} \\
& \left\langle\chi_{\mathrm{i}}^{(2)}\right\rangle_{2}=\frac{\alpha^{2} \sigma}{\pi} \cdot \frac{\mu_{a b}^{(3)} \mu_{b c}^{(1)} \mu_{c a}^{(2)}}{\left[\left(\omega_{2}-\omega_{c a}^{0}\right)+\alpha\left(\omega_{b a}^{0}-\omega_{3}\right)+i\left(\alpha \Gamma_{a b}-\Gamma_{a c}\right)\right]} \\
& \quad \times \frac{1}{\left[\omega_{2}-\omega_{c a}^{0}-\left(\Gamma_{c a}+\alpha \sigma\right)\right]\left[\omega_{2}-\omega_{c a}^{0}-i\left(\Gamma_{c a}-\alpha \sigma\right)\right]} .
\end{aligned}
$$

In this expression one of the resonance denominators is not affected by inhomogeneous parameters, and line narrowing should occur with a width:

$$
\Gamma=\Gamma_{a c}+|\alpha| \Gamma_{a b}
$$

Actually, $\alpha<0$ means strict anticorrelation of excited states, i.e., a positive energy shift of level $b$ is related to a negative shift of level $c$. The case of correlation $(\alpha>0)$ should yield no line narrowing in type I processes. However certain situations might result in anticorrelation. For example, a large dipole moment change on excitation could be in opposite direction for the $a-b$ and $a-c$ transitions. The line narrowing experiment in the type I process then could serve as a probe for anticorrelation. ${ }^{35}$

In the type II processes the susceptibility gives rise to three poles in addition to those from the distribution function: $p_{1}=i \sigma ; p_{2}=-i \sigma ; p_{3}=\omega_{1}-\omega_{b a}^{0}-i \Gamma_{a b} ; p_{4}=\left(\omega_{2}\right.$ $\left.-\omega_{c a}^{0}+i \Gamma_{a c}\right) / \alpha$; and $p_{5}=\left(\omega_{3}-\omega_{c b}^{0}+i \Gamma_{b c}\right) /(\alpha-1)$. As is obvious from $F i g$. 7 , we need only consider the residues $\left(R_{i}\right)$ at the poles $p_{1}, p_{4}$, and $p_{5}$. These are:

$$
\begin{aligned}
R_{1}= & \frac{1}{2 \pi i} \frac{\mu_{c a}^{(2)} \mu_{a b}^{(1)} \mu_{b c}^{(3)}}{\left[\omega_{b a}^{0}-\omega_{1}+i\left(\Gamma_{c b}+\sigma\right)\right]\left[\omega_{c a}^{0}-\omega_{2}-i\left(\Gamma_{a c}-\alpha \sigma\right)\right]}\left\{1+i \frac{\Gamma_{b c}-\Gamma_{a b}-\Gamma_{a c}}{\left[\omega_{c b}^{0}-\omega_{3}-i\left(\Gamma_{b c}+\sigma-\alpha \sigma\right)\right]},\right. \\
R_{4}= & \frac{-\alpha^{2} \sigma}{\pi} \frac{\mu_{c a}^{(2)} \mu_{a b}^{(1)} \mu_{b c}^{(3)}}{\left[\alpha\left(\omega_{c b}^{0}-\omega_{3}\right)+(\alpha-1)\left(\omega_{2}-\omega_{c a}^{0}\right)-i\left(\alpha \Gamma_{c b}-(\alpha-1) \Gamma_{c a}\right)\right]} \\
& \times \frac{1}{\left[\omega_{2}-\omega_{c a}^{0}+i\left(\Gamma_{c a}+\alpha \sigma\right)\right]\left[\omega_{2}-\omega_{c a}^{0}+i\left(\Gamma_{c a}-\alpha \sigma\right)\right]}, \\
R_{5}= & \frac{\sigma(\alpha-1)^{2}}{\pi} \cdot\left\{\frac{1}{\left[(\alpha-1)\left(\omega_{b c}^{0}-\omega_{1}\right)+\left(\omega_{3}-\omega_{c b}^{0}\right)+i\left[(\alpha-1) \Gamma_{a b}+\Gamma_{b c}\right)\right]}\right. \\
& \left.-\frac{1}{\left[\left(\omega_{c a}^{0}-\omega_{2}\right)(\alpha-1)+\alpha\left(\omega_{3}-\omega_{c b}^{0}\right)+i\left(\alpha \Gamma_{b c}-(\alpha-1) \Gamma_{a c}\right)\right]}\right\} \\
& \times \frac{\mu_{c a}^{(2)} \mu_{a b}^{(1)} \mu_{b c}^{(3)}}{\left[\omega_{3}-\omega_{c b}^{0}+i\left(\Gamma_{b c}+(\alpha-1) \sigma\right)\right]\left[\omega_{3}-\omega_{c b}^{0}+i\left(\Gamma_{b c}+(1-\alpha) \sigma\right)\right]} .
\end{aligned}
$$

Again, the residues at the poles from the distribution function are not line narrowed, while the other residues contain line narrowed resonances. The nature of the resonance linewidths depend on the sign and magnitude of $\alpha$ as follows [see Figs. 7(c) $-7(\mathrm{e})$ ]:

$$
\begin{aligned}
& \left\langle\chi_{\mathrm{II}}^{(2)}\right\rangle=2 \pi i R_{1}, \quad \alpha<0, \\
& \left\langle\chi_{I I}^{(2)}\right\rangle=2 \pi i\left(R_{1}+R_{4}\right), \quad 0<\alpha<1 \text {, } \\
& \left\langle\chi_{11}^{(2)}\right\rangle=2 \pi i\left(R_{1}+R_{4}+R_{5}\right), \quad 1<\alpha .
\end{aligned}
$$

In the case of anticorrelation $(\alpha<0)$ no line narrowing should be possible, while in the case of correlation $(\alpha>0)$ line narrowing occurs. When the inhomogeneous width is much larger than the homogeneous parameters, $\sigma \gg\left(\Gamma_{a b}, \Gamma_{a c}, \Gamma_{b c}\right)$, all line narrowed signals are predicted to be single Lorentzian in shape. For the three scan modes discussed in the previous section we expect the widths of these resonances shown in Table II. The results for type II, $\alpha>1$ are independent of $\Gamma_{a c}$ although both residues $R_{4}$ and $R_{5}$ contain $\Gamma_{a c}$ in their resonances.
These contributions, however, cancel out. This is obvious from Fig. $7(\mathrm{e})$, since the line narrowing contributions from the residue $R_{3}$ alone must give the same result. All three scan modes are predicted to yield the same information since the resulting widths always contain the same linear combination of the homogeneous

TABLE II. Predicted linewidths for various scan modes in the presence of inhomogeneous broadening.

\begin{tabular}{llll}
\hline \hline & \multicolumn{3}{c}{ Fixed frequency } \\
\cline { 2 - 4 } Type & $\omega_{1}$ & $\omega_{2}$ & $\omega_{3}$ \\
\hline I; $\alpha<0$ & $\frac{\Gamma_{a b}+|\alpha| \Gamma_{a b}}{1+|\alpha|}$ & $\frac{\Gamma_{a b}+|\alpha| \Gamma_{a b}}{|\alpha|}$ & $\Gamma_{\alpha c}+|\alpha| \Gamma_{a b}$ \\
II; $0<\alpha<1$ & $\alpha \Gamma_{b c}+(1-\alpha) \Gamma_{a c}$ & $\frac{\alpha \Gamma_{b c}+(1-\alpha) \Gamma_{a c}}{\alpha}$ & $\frac{\alpha \Gamma_{b c}+(1-\alpha) \Gamma_{a c}}{1-\alpha}$ \\
II; $\alpha>1$ & $\Gamma_{b c}+(\alpha-1) \Gamma_{a b}$ & $\frac{\Gamma_{b c}+(\alpha-1) \Gamma_{a b}}{\alpha}$ & $\frac{\Gamma_{b c}+(\alpha-1) \Gamma_{a b}}{\alpha-1}$ \\
\hline
\end{tabular}


parameters, and $\alpha$ was already obtained as the ratio of the inhomogeneous widths. Finally, no problems arise in the special case $\Gamma_{b c}=\Gamma_{a c}+\Gamma_{a b}$ although the resonance at $\omega_{a b}$ and consequently the pole $p_{5}$ does not occur since $R_{5}$ is then identically zero.

The integration over a Gaussian inhomogeneous distribution can be accomplished by first decoupling the denominators in the form:

$$
\frac{1}{a b}=\frac{1}{b-a}\left(\frac{1}{a}-\frac{1}{b}\right) \text {, }
$$

then using the integral representation for the complex error function $w(s z)$ [where $s=\operatorname{sign}(\operatorname{Im} z)$ ]:

$$
\int_{-\infty}^{+\infty} \frac{e^{-t^{2}} d t}{z-t}=-i \pi s w(s z)
$$

to obtain the following results in which $G$ indicates Gaussian averaging:

$$
\begin{aligned}
\left\langle\chi_{s}^{(2)}\right\rangle_{G}= & \frac{i \mu_{a b}^{(3)} \mu_{b c}^{(1)} \mu_{c a}^{(2)}(\pi / 2)^{1 / 2}}{\sigma B-\alpha A]} \\
& \times\{w(A / \sigma \sqrt{2})-S(\alpha) w(B /|\alpha| \sigma \sqrt{2})\}, \\
\left\langle\chi_{\mathrm{II}}^{(2)}\right\rangle_{G}= & \frac{i \mu_{a b}^{(3)} \mu_{b c}^{(1)} \mu_{c e}^{(2)}(\pi / 2)^{1 / 2}}{\sigma}\left\{\frac{1}{E-(\alpha-1) C}\right. \\
& \times[w(C / \sigma \sqrt{2})-S(1-\alpha) w(E /|1-\alpha| \sigma \sqrt{2})] \\
& -\frac{1}{\alpha E-(\alpha-1) D}[S(\alpha) w(D /|\alpha| \sigma \sqrt{2}) \\
& -S(1-\alpha) w(E /|1-\alpha| \sigma \sqrt{2})]\},
\end{aligned}
$$

with the abbreviations: $A=\omega_{b c}^{0}-\omega_{3}+i \Gamma_{a b} ; B=\omega_{c a}^{0}$ $-\omega_{2}+i \Gamma_{c a} ; C=\omega_{b a}^{0}-\omega_{1}+i \Gamma_{a b} ; D=\omega_{c a}^{0}-\omega_{2}-i \Gamma_{a c} ;$ and $E=\omega_{c b}^{0}-\omega_{3}-i \Gamma_{b c}$. Some simulated line shapes, both for Lorentzian and Gaussian distributions, are shown in Fig. 8. It is obvious from these curves that Gaussian and Lorentzian distributions give essentially the same narrowed line shape even when the homogeneous and inhomogeneous widths are of the same order of magnitude.

The results of the previous paragraph encouraged us to perform the average of $\chi^{(2)}$ [Eq. (27)] with a two dimensional distribution function of the type in Eq. (28) using Lorentzian bell functions $g_{2}[\mathrm{Eq}$. (32)]. In this case the integration could be carried through analytically in two consecutive contour integrations. During these two steps at least one of the two inhomogeneous width parameters $\sigma_{1}$ and $\sigma_{2}$ is added to each of the resonance denominators. Some interesting situations that may arise in molecular problems are: (1) The system is governed by the inequality $\sigma_{1} \gg \Gamma>\sigma_{2}$. This case closely resembles the limit of perfect correlation. Here $\Gamma$ is some linear combination of the homogeneous width parameters (such as those in Table II) depending on the angle $\phi$ by which the $(x y)$ and $(\xi \eta)$ axis systems are rotated. The narrowed lines will have width $\boldsymbol{\Gamma}+\sigma_{2}$, implying essentially homogeneous linewidths. The criteria for the corresponding residues to occur are

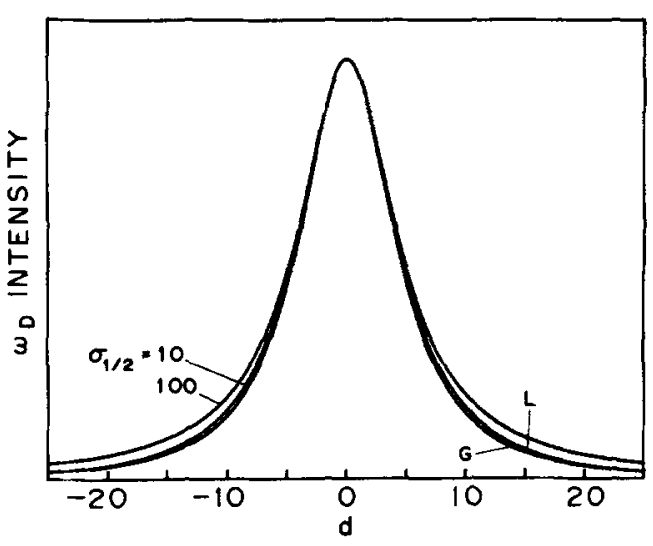

FIG. 8. Simulated narrowed line with Gaussian (G) and Lorentzian $(L)$ distribution functions as indicated. parameters were $\Gamma_{a b}=\Gamma_{a c}=\Gamma_{b c}=5$, type II resonance, $\alpha=1$. The FWHM of the inhomogeneous distribution was set at 10 and 100 as indicated.

Type II: $0<\phi<\pi / 2$; Type I: $-\pi / 2<\phi<0$.

The longer half-axis of the distribution, in our case $\sigma_{1}$, must have the same orientation as the correlation line in the strict correlation limit. (2) The system is defined by the inequality $\sigma_{1} \gg \sigma_{2} \geqslant \Gamma$. Again the narrowed lines show width $\sigma_{2}+\Gamma$ under the same conditions as in case (1), but now the correlation coefficient is probed rather then the homogeneous parameters. The inhomogeneously broadened widths of the two states are

$$
\begin{aligned}
& \Delta_{x}=|\cos \phi| \sigma_{1}+|\sin \phi| \cdot \sigma_{2}, \\
& \Delta_{y}=|\sin \phi| \sigma_{1}+|\cos \phi| \cdot \sigma_{2} .
\end{aligned}
$$

Thus the determination of an absolute lower bound to the correlation coefficient should be possible. (3) The system is governed by the inequality $\sigma_{1} \simeq \sigma_{2} \gg \Gamma$. This situation corresponds to low correlation, and certainly line narrowing is not possible.

\section{EFFECT OF EXCITED STATE POPULATION}

The calculations and discussion so far were carried out on the assumption that no excited state populations can initiate the sum and difference frequency generation process. This was justified by the fact that significant population contributions to these processes are built up from third and higher order in the iterative integration procedure if the starting population is all in the ground date. Of course coherence between excited levels requires these levels to be populated to some extent. Furthermore, we may expect that populations formed early in the pulse envelope through higher order processes, feeding and by dephasing effects (i.e., incoherent populations) will contribute to the coherent signal. Each state which acts as a bottleneck in the relaxation towards thermal equilibrium will serve as an initiating state for the evolution of the density operator. For example, the vibrationless level of the lowest excited electronic state would be expected to be long-lived enough to initiate new nonlinear processes in this manner. If the decay of the bottleneck level is negligible with respect to the other time constants in the system: i.e., if $\gamma_{b b} \ll \Gamma_{i j}$ for all $i$ and $j$, then the 


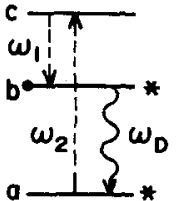

(a)

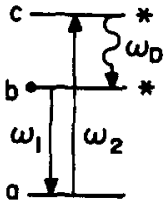

(b)

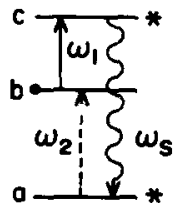

(c)

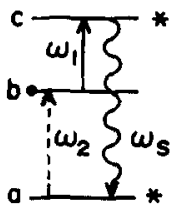

(d)
FIG. 9. Diagrammatic representation of the fully resonant contributions to $X^{(2)}$ from an initial population in level $b$. For discussion see the text.

feeding terms may again be neglected and the calculation of the density matrix is carried out using the same techniques as described in Sec. II. In case this inequality is not met the time evolution of the starting population would have to be included.

We treat the case where the middle state of the three level system initiates the nonlinear process. The diagram for the time development of the density matrix is readily obtained from Fig. 1 by the cyclic permutation of the indices $(a, b, c)$ to $(b, c, a)$. The nondiagonal terms of interest are $\rho_{c b}^{(2)}$ and $\rho_{b a}^{(2)}$ with only one path contributing, and $\rho_{a c}^{(2)}$ with two paths. The corresponding susceptibilities are, therefore, of type $I$ for the first two matrix elements and of type II for the latter. The level diagrams corresponding to these four paths are given in Fig. 9 which is readily obtained from Fig. 2 by shifting the highest level below level $a$ and relabeling the levels in order $a, b, c$ from bottom to top. The arrows representing the field components remain attached to the levels. However, in Figs. 9(a) and 9 (b) $\omega_{1}$ and $\omega_{2}$ were interchanged to emphasize the similarities between the diagrams in Figs. 2 and 9 .

A comparison of the diagrams for the two initial conditions reveals, that the excited state population will make a contribution to all three processes discussed in Sec. 2. Diagram 9(a) makes a contribution to the type I difference frequency generation ${ }^{26}$ but with different time ordering. A time resolved experiment should, therefore, allow these contributions to be distinguished. The difference frequency generation between two excited levels, the type II processes 2(c) and 2(d), will get an additional term from diagram $9(\mathrm{~b})$. The latter is, however, of type I and shows consequently only one time ordering, namely that of diagram 2 (c).

The most interesting case is the type II sum frequency generation $9(c)$ and $9(d)$ induced by the initial population in level $b$. It shows the extra resonance:

$$
\begin{aligned}
\chi^{(2)}[9(\mathrm{c}) \text { and } 9(\mathrm{~d})]= & -\rho_{b b}^{0} \cdot \frac{\mu_{b a}^{(1)} \mu_{c b}^{(2)} \mu_{a c}^{(3)}}{\left[\omega_{c b}-\omega_{1}-i \Gamma_{c b}\right]\left[\omega_{b a}-\omega_{2}-i \Gamma_{b a}\right]} \\
& \times\left\{1+i \frac{\Gamma_{c a}-\Gamma_{c b}-\Gamma_{b a}}{\left[\omega_{c a}-\omega_{s}-i \Gamma_{c a}\right]}\right\} . \quad \text { (46) }
\end{aligned}
$$

In the absence of pure dephasing the numerator of the additional resonance reduces to the inverse lifetime of the level $b$ : i.e., $\left(-\Gamma_{c a}+\Gamma_{c b}+\Gamma_{b a}\right)-\gamma_{b b}$. Since we assumed this parameter to be very small, the $\omega_{c a}$ resonance will exhibit a DICE effect. In principle, this DICE resonance occurs even when there is no pure dephasing because of the finite value of $\gamma_{b b}$ derived from spontaneous emission or radiationless processes. The discussion of the diagrams $9(a)-9$ (d) can easily be extended to their line narrowing characteristics. Adopting the substitution $\omega_{b a}=\omega_{b a}^{0}+x$ and $\omega_{c a}=\omega_{c a}^{0}+\alpha x$ as in Sec. $\mathrm{V}$, the pole arguments can be applied in an analogous manner. It turns out that all diagrams can be line narrowed and that the conditions for $\alpha$ are complementary in the case 9(a) and 9(b) and the case 9(c) and $9(\mathrm{~d})$ : The difference frequency diagrams $9(\mathrm{a})$ and 9 (b) can be line narrowed with $\alpha>1$, while the sum frequency line narrowing requires $\alpha<1$. These conditions are different from those for the diagrams 2(a)2(d) and could be used to gain additional information about the correlations within the various inhomogeneous distributions.

\section{CONCLUSIONS}

Our investigation of the spectroscopic properties of $\chi^{(2)}$ under fully resonant conditions lead to the following conclusions:

(i) Fully resonant sum and difference frequency generation are both possible processes.

(ii) There exist two mechanisms for difference frequency generation, namely: difference frequency generation I having resonances on transitions from the initial state; and difference frequency generation II having a resonance on a transition between two initially unpopulated states.

(iii) If the system shows no inhomogeneous broadening of its transitions the line shapes of the resonances contain possible information about $\Gamma_{a b}$ and $\Gamma_{a c}$ depending on which in-going frequency is scanned.

(iv) In the case of difference frequency generation II there are circumstances under which the line shape also depends on $\Gamma_{b c}$, the coherence decay parameter for the pair of excited levels. A dephasing induced coherent emission (DICE) effect is predicted in difference frequency generation II.

(v) When the inhomogeneous distributions of the $a-b$ and $a-c$ transitions have correlation factors $q \neq 0$, all three processes contain line narrowing capabilities which are partly complementary. If $q>0$, line narrowing should occur in difference frequency generation II, while sum frequency generation and difference frequency generation I processes should yield narrowed lines only in the case $q<0$.

(vi) All the foregoing resonances may arise as a result of excited state populations being formed from which nonlinear processes can be initiated. Population in level $b$ will give peaks that could be confused with the extra (DICE) resonance.

This study has shown that a full exploration of $\chi^{(2)}$ resonant processes is likely to yield new spectroscopic information. In addition measurements of dynamical parameters such as pure dephasing should be possible. The present work through focused on $\chi^{(2)}$ has obvious implications in $\chi^{(3)}$ spectroscopy. The same pathways giving line narrowing as described herein, and DICE 
effects, lead to generation of coherent light in third order processes. In fact the two pathways giving rise to $\rho_{b c}^{(2)}$, and hence to difference frequency generation DICE, are the origin of the two terms that give the extra CSRS resonances previously described. ${ }^{18,22}$ The present treatment of the principles determining the line shapes for the inhomogeneous cases is therefore useful in both $\chi^{(3)}$ and $\chi^{(2)}$ spectroscopy. However the description is simpler, there being one fewer resonance denominator, for the $\chi^{(2)}$ response.

${ }^{1}$ F. C. McGurk, T. G. Schmalz, and W. H. Flygare, Adv. Chem. Phys. 25, 1 (1974).

${ }^{2}$ V. S. Letokhov, in High Resolution Laser Spectroscopy, Topics in Applied Physics, edited by K. Shimoda (Springer, Berlin, 1976), Vol. 13.

${ }^{3}$ V. S. Letokhov and V. P. Chebotayev, Nonlinear Laser Spectroscopy (Springer, Berlin, 1977).

${ }^{4}$ R. A. McFarlane, W. R. Bennett, Jr., and W. E. Lamb, Jr. , Appl. Phys. Lett. 2, 189 (1963).

${ }^{5}$ A. Szöke and A. Javan, Phys. Rev. Lett. 10, 521 (1963).

${ }^{6}$ C. Freed and A. Javan, Appl. Phys. Lett. 17, 53 (1970).

${ }^{7}$ F. Biraben, B. Cagnac, and G. Grynberg, Phys. Rev, Lett. 32, 643 (1974).

${ }^{8}$ T. W. Hänsch, K. Harvey, G. Meisel, and A. L. Schawlow, Opt. Commun. 11, 50 (1974).

${ }^{9}$ M. D. Levenson and N. Bloembergen, Phys. Rev. Lett. 32, 645 (1974).

${ }^{10} \mathrm{C}$. Wieman and T. W. Hänsch, Phys. Rev. Lett. 36, 1170 (1976).

${ }^{11}$ F. Gelbwachs, P. F. Jones, and J. E. Wessel, Appl. Phys. Lett. 27, 551 (1975).

${ }^{12}$ R. E. Smalley, D. H, Levy, and L. Wharton, J. Chem. Phys. 64, 3266 (1976).

${ }^{13}$ D. H. Levy, L. Wharton, and R. E. Smalley, Acc. Chem. Res. 10, 134 (1977).

${ }^{14}$ D. H. Levy, Annu. Rev. Phys. Chem. 31, 197 (1980).

${ }^{15}$ A. Szabo, Phys. Rev. Lett. 25, 924 (1970); 27, 323 (1971).

${ }^{16} \mathrm{D}$. A. Wiersma, Adv. Chem. Phys. 47, 421 (1981).

${ }^{17}$ P. L. DeCola, F. R. Andrews, R. M. Hochstrasser, and $H$. P. Trommsdorff, J. Chem. Phys, 73, 4695 (1980).

${ }^{18} \mathrm{~J}$. R. Andrews and R. M. Hochstrasser, Chem. Phys. Lett. 83,427 (1981).

${ }^{19}$ J. R. Andrews, R. M. Hochstrasser, and H. P. Trommsdorff, Chem. Phys, 62, 87 (1981).

${ }^{20}$ (a) A. R. Bodgan, Y. Prior, and N. Bloembergen, Opt. Lett. B, 82 (1981); (b) N. Bloembergen, H. Lotem, and R. T. Lynch,

Jr. , Ind. J. Pure Appl. Phys. 16, 151 (1977).

${ }^{21} R$. M. Hochstrasser and H. P. Trommsdorff, Acc. Chem.

Res. (in press).

${ }^{22}$ J. R. Andrews and R. M. Hochstrasser, Chem. Phys. Lett. 82,381 (1981).

${ }^{23}$ J. L. Oudar and Y. R. Shen, Phys. Rev. A 22, 1141 (1980).
${ }^{24}$ S. A. L. Druet, J. P. E. Taran, and Ch. J. Borde, J. Phys. 40, 841 (1979).

${ }^{25}$ S. A. L. Druet, J.-P. E. Taran, and Ch. J. Borde, J. Phys. (Paris) 41, $183(1980)$.

${ }^{26}$ J. H. Lee, J. J. Song, M. A. F. Scarparo, and H. D. Levenson, Opt. Lett. 5, 196 (1980).

${ }^{27}$ E. J. Heilweil, R. M. Hochstrasser, and H. Souma, Opt. Commun. 35, 227 (1980).

${ }^{28} \mathrm{H}$. Souma, E. J. Heilweil, and R. M. Hochstrasser, J. Chem. Phys. 76, 5693 (1982).

${ }^{29}$ R. M. Hochstrasser and G. R. Meredith, J. Chem. Phys. 67, 1273 (1977); Pure Appl. Chem. 50, 759 (1978).

${ }^{30}$ T. F. Heinz, C. K. Chen, D. Richard, and Y. R. Shen, Phys. Rev. Lett. 48, 478 (1982).

${ }^{31} \mathrm{~N}$. Bloembergen, Nonlinear Optics (Benjamin, New York, $1965)$.

${ }^{32}$ T. K. Yee and T. K, Gustafson, Phys. Rev. A 18, 1597 (1978).

${ }^{33}$ It is interesting to note that the linewidths obtained in this way can be much narrower than the width of the state with which the scanned ingoing beam is in resonance. Taking the type I difference frequency generation as an example, the state $c$ will be higher in energy than the state $b$ [compare Fig. 2(b)], and $\Gamma_{a c} \gg \Gamma_{a b}$. Scanning the laser $\omega_{2}$ over the resonance $\omega_{a a}$ having the width $\Gamma_{a c}$ will yield a signal with the much smaller width $\Gamma_{a b}$.

${ }^{34}$ The correlation coefficient is defined as $q=\langle x y\rangle /(\langle x x\rangle\langle y y\rangle)^{1 / 2}$, where the brackets indicate the average over the distribution. For the two dimensional Gaussian distribution this result is

$$
q_{G}=\frac{C S\left(\sigma_{1}^{2}-\sigma_{2}^{2}\right)}{\sqrt{\left(S^{2} \sigma_{1}^{2}+C^{2} \sigma_{2}^{2}\right)\left(C^{2} \sigma_{1}^{2}+S^{2} \sigma_{2}^{2}\right)}},
$$

with $C=\cos \phi$ and $S=\sin \phi$. Strictly speaking, the correlation coefficient cannot be calculated for the two dimensional Lorentzian distribution since $\langle x x\rangle,\langle y y\rangle$, and $\langle x y\rangle$ diverge. The distribution function may, however, be modified in the following way:

$$
\tilde{g}_{L}(x, \sigma)=\frac{\sigma / \pi}{x^{2}+\sigma^{2}} \cdot \frac{1}{1+x^{2} \cdot b^{2}} .
$$

In the limit $b \rightarrow 0$ this function again becomes the Lorentzian $g_{L}$. The averages $\langle x x\rangle$, etc. may now be obtained using the two-dimensional distribution $\tilde{g}_{L}\left(\xi, \sigma_{1}\right)=\tilde{g}_{L}\left(\pi, \sigma_{2}\right)$ and $q$ may be calculated. After taking the limit $b \rightarrow 0$ one obtains:

$$
q_{L}=\frac{C S\left(\sigma_{1}-\sigma_{2}\right)}{\sqrt{\left(S^{2} \sigma_{1}+C^{2} \sigma_{2}\right)\left(C^{2} \sigma_{1}+S^{2} \sigma_{2}\right)}} .
$$

Obviously for both Gaussian and Lorentzian distributions $q$ vanishes with either $\cos \phi$ or $\sin \phi$ being zero. The other limit $|q|=1$ is obtained if $\sigma_{1}$ or $\sigma_{2}$ vanishes.

${ }^{35}$ The result for $\alpha>0$ can also be obtained from the poles $p_{2}$, $p_{3}$, and $p_{4}$. Although the latter two individually give a line narrowing term, the complete result is certainly not line narrowed. The line narrowing contributions cancel out in this case. Care has to be taken, therefore, if a sum of line narrowed terms appear. 